\title{
Minimally invasive techniques and indocyanine angiography in anterior abdominal wall reconstruction after multiple laparotomies
}

\author{
Tomasz Buczek $^{1}$, Wiesław Tarnowski ${ }^{2}$, Marcin Kuriata ${ }^{1}$, Maciej Śmietański ${ }^{1}$ \\ ${ }^{1}$ Department of General Surgery, Puck Hospital, Puck, Poland \\ ${ }^{2}$ Department of General, Oncological and Digestive Tract Surgery, Medical Centre of Postgraduate Education, Orłowski Hospital, \\ Warsaw, Poland
}

Videosurgery Miniinv 2018; 13 (3): 412-416

DOI: https://doi.org/10.5114/wiitm.2018.75999

\begin{abstract}
Anterior abdominal wall reconstruction surgery in patients who have undergone multiple laparotomies is associated with a high risk of complications. For this reason, minimally invasive surgery techniques are used to avoid abdominal compartment syndrome, to enhance wound healing and to reduce postoperative pain and blood loss. This paper presents a case of a patient who was admitted to the Department of General Surgery in Puck Hospital as a result of extensive anterior abdominal wall deformation with an end descending colostomy. A single-stage procedure was performed: specifically, digestive tract reconstruction followed by anterior abdominal wall reconstruction using intraoperative angiography of indocyanine green in infrared light.
\end{abstract}

Key words: strip binding technique, anterior wall reconstruction, minimally invasive techniques, ICG, Ramirez.

\section{Introduction}

This paper presents a case of a patient who was admitted to the Department of General Surgery in Puck Hospital as a result of extensive anterior abdominal wall deformation with an end descending colostomy. In the past, the patient had undergone multiple laparotomies in the course of Crohn's disease. A single-stage procedure was performed: specifically, digestive tract reconstruction followed by anterior abdominal wall reconstruction was performed using intraoperative angiography of indocyanine green in infrared light.

\section{Case report}

A 34-year-old patient with a body mass index (BMI) of $28 \mathrm{~kg} / \mathrm{m}^{2}$ and extensive post-laparotomy an- terior abdominal wall deformation with an end descending colostomy (Photo 1) was admitted to the Department of General Surgery in order to restore the continuity of the gastrointestinal tract and anterior abdominal wall reconstruction. In the past, the patient had undergone 14 laparotomies because of Crohn's disease (multiple colon resections complicated with colon perforation and anastomosis leakage). Intraoperative examination revealed a loss of fascia with total dimensions of $23 \times 14 \mathrm{~cm}$. Adhesiolysis was followed by gastrointestinal tract reconstruction using a ф $29 \mathrm{~mm}$ circular stapler. Subsequently, Ramirez SBT (Strip Binding Technique) component separation using a sublay mesh was performed; the rectus sheath was opened from the xiphoid process to $3 \mathrm{~cm}$ below the umbilicus (Photo 2). The external oblique muscle insertion was cut off from the ribs to the um- 


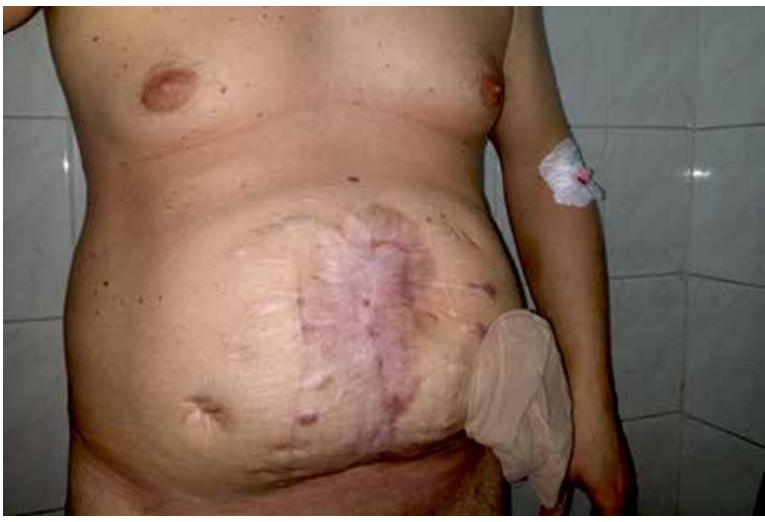

Photo 1. Patient underwent multiple laparotomies in the course of Crohn's disease. Extensive post-laparotomy anterior abdominal wall deformation with an end descending colostomy

bilicus; the space between the oblique muscles (external and internal bilaterally) was dissected (Photo 3). The peritoneum was reconstructed with continuous absorbable suture 0. Macroporous mesh Parietene $15 \times 35 \mathrm{~cm}$ (Medtronic) was placed in the sublay position with three strips according to the Strip Binding Technique; three mesh strips that were $1.5 \mathrm{~cm}$ wide were drawn under the rectus muscle through the aponeurosis, pulled medially and fixed to the anterior rectus sheath. To strengthen the abdominal wall in the post-stoma area, the mesh was expanded and placed in the underlay position. The anterior rectus sheath and linea alba were reconstructed using Prolene continuous suture 0 . The bridging technique was performed on a length of $12 \mathrm{~cm}$ and a width of $3 \mathrm{~cm}$ (Photo 4). To assess the blood supply to cutaneo-subcutaneous wound edges, indocyanine green angiography in infrared light was performed (Karl Storz, Image1 S, Full HD in the configuration of the ICG; dye Verdye $5 \mathrm{mg} / \mathrm{ml}$ Diagnostic Green). $1 \mathrm{ml}$ of Verdye was administered intravenously by the anesthesiologist. Blood supply to the cutaneo-subcutaneous flaps was assessed using infrared light from a laparoscopic camera. The tissue well supplied with blood glowed green in infrared light, whereas ischemic tissue was not illuminated. The cut-off point was defined and marked on a green light border. One Redon drain was placed on the implant, and two drains were placed in the subcutaneous tissue. The surgery lasted $2 \mathrm{~h}$ and $40 \mathrm{~min}$. The postoperative course was uneventful. The patient was discharged on the sixth day after surgery (Photo 5). A follow-up examination in the

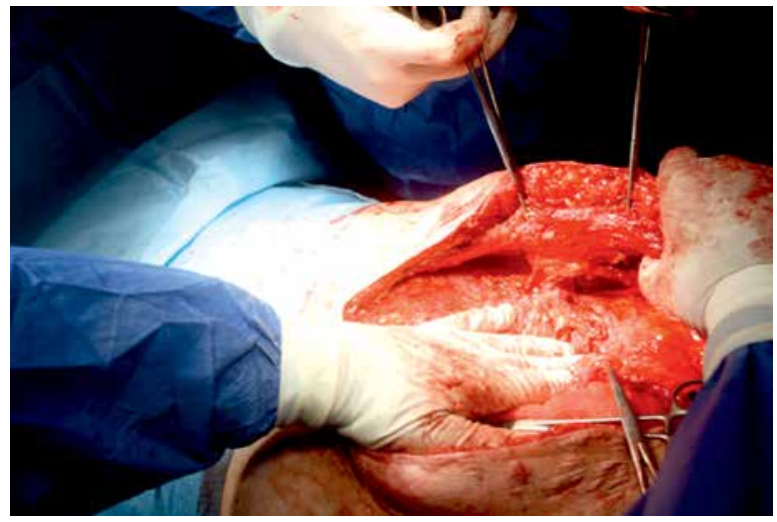

Photo 2. Dissection of the rectus muscle sheath with its perforator from the level of the xiphoid process up to approximately $3 \mathrm{~cm}$ below the umbilicus

third month after surgery revealed complete wound healing, correct function of the gastrointestinal tract and no hernia recurrence (Photo 6).

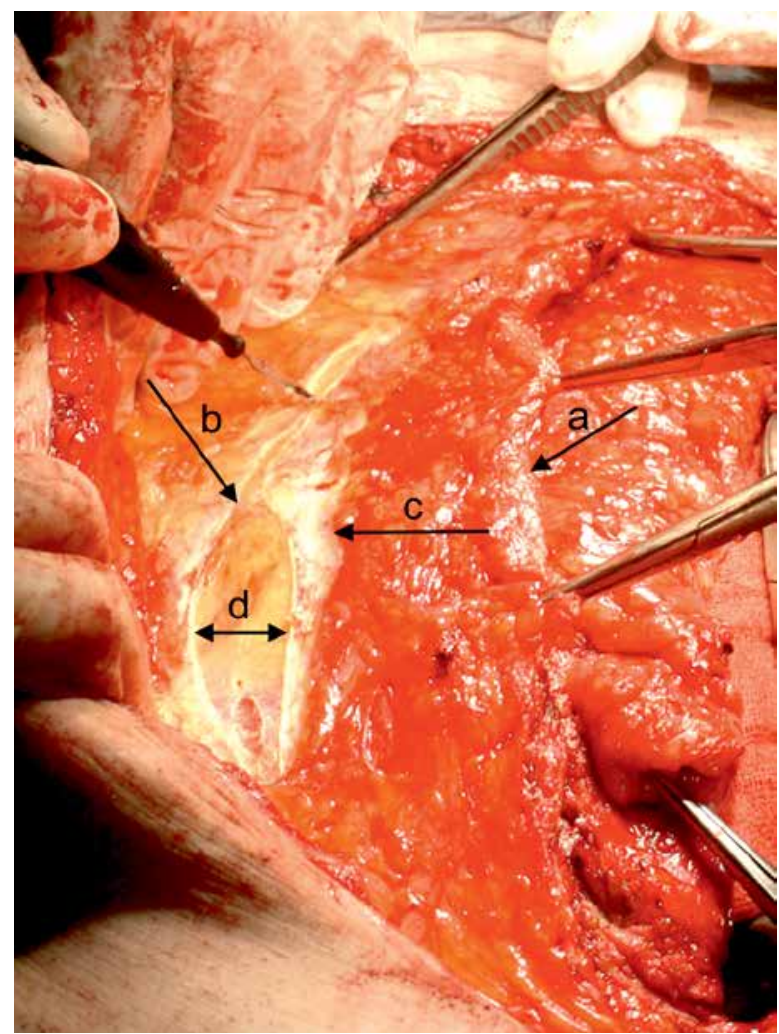

Photo 3. Dissecting the space between the oblique muscles: $a$ - anterior rectus sheath, $b$ - aponeurosis of internal oblique, $c$ - abdominal external oblique muscle insertion, $d$ - distance obtained after insertion of the oblique muscle cutoff 


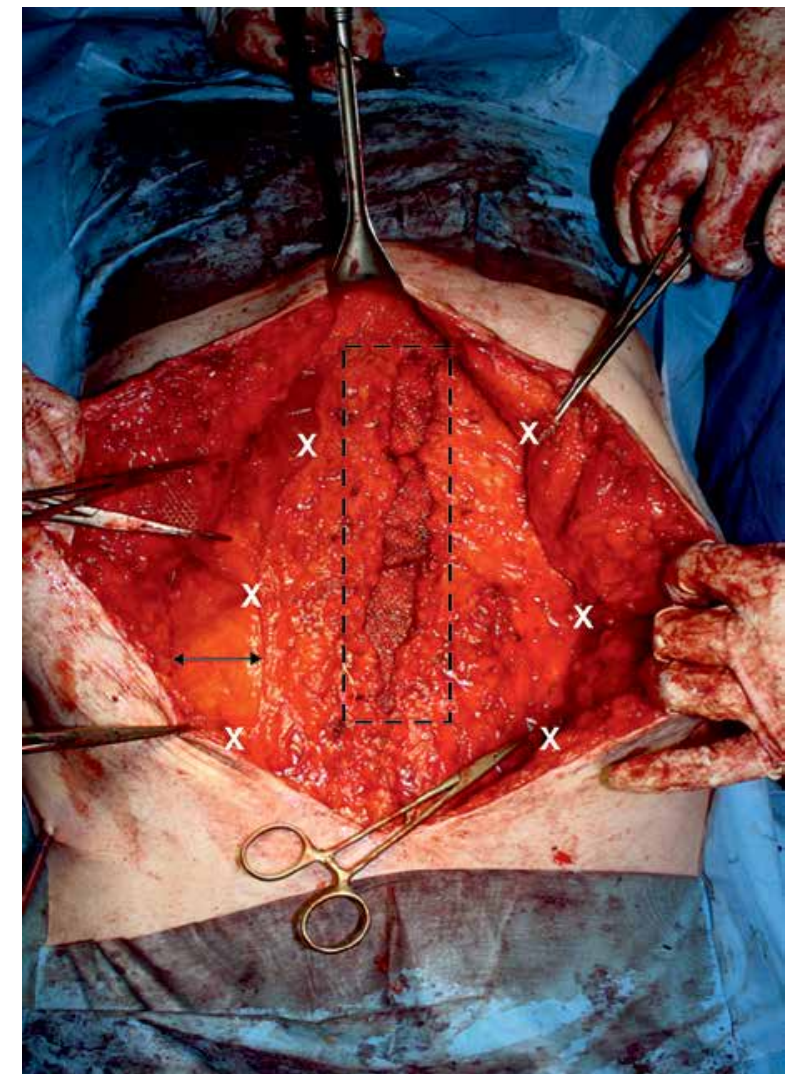

Photo 4. The bridging technique. X-points where strips are fixed to the anterior rectus sheath

Border - bridging area; arrow - distance obtained after cutoff of the external oblique muscle insertion.

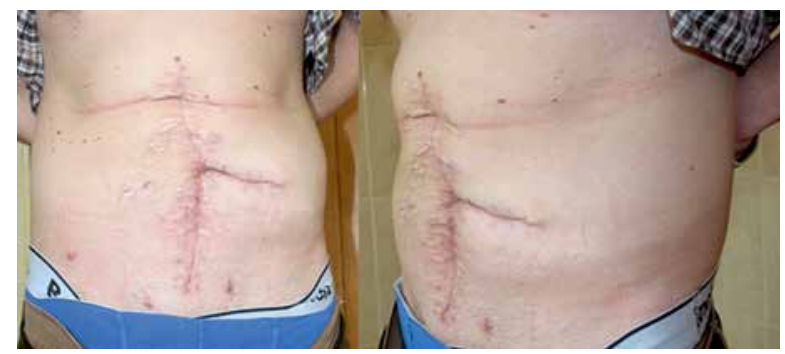

Photo 6. Seventy-fifth day after surgery

\section{Discussion}

Anterior abdominal wall reconstruction surgery in patients who have undergone multiple laparotomies is associated with a high risk of complications. These complications include intestine perforation and bleeding during adhesiolysis and, in the postoperative course, abdominal compartment syndrome, skin necrosis, seromas and surgical site infection. For this reason, minimally invasive surgery techniques are used to avoid abdominal compartment

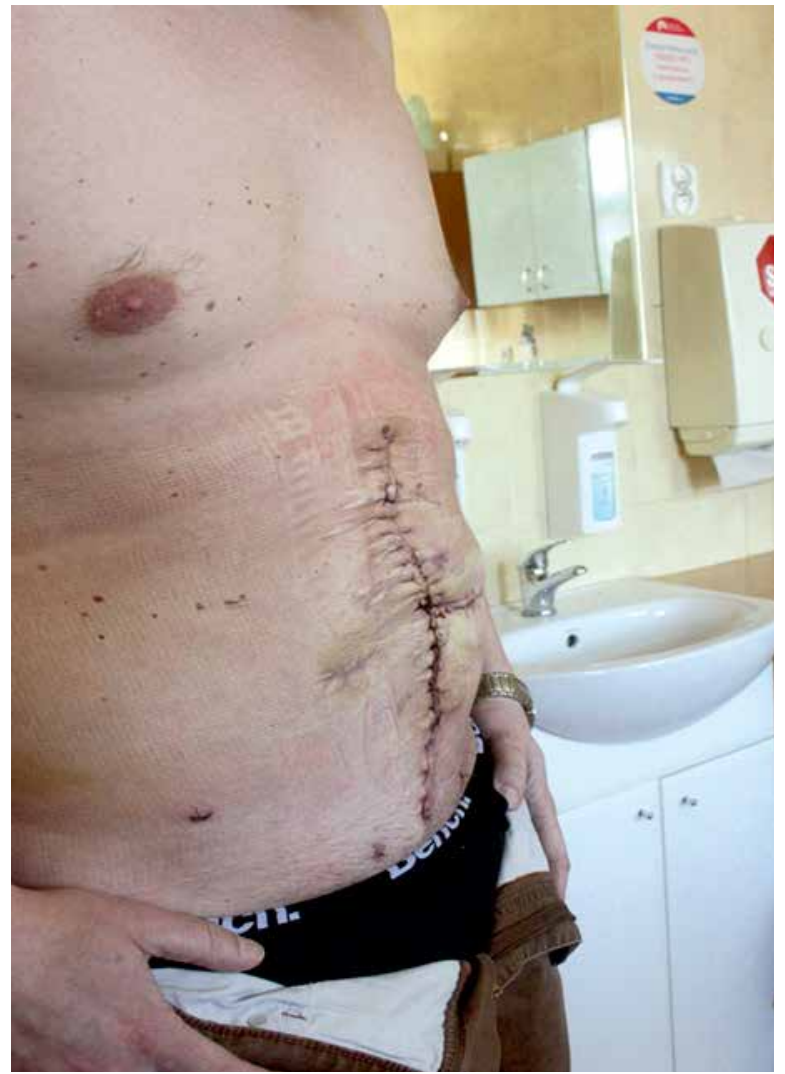

Photo 5. Sixth day after surgery

syndrome, to enhance wound healing and to reduce postoperative pain and blood loss.

Minimally invasive surgery not only means a smaller skin incision; it is also a set of techniques that reduces perioperative trauma and leads to faster recovery and patient discharge. The use of these methods, including some principles of the ERAS protocol, in the case described in this report enables these aims to be achieved [1].

Dissecting anatomical layers reduces intraoperative blood loss, which is an independent factor of hernia recurrence. The mechanism of action is associated with blood plasma protein loss (resulting from increased intraoperative bleeding) that impairs healing. For this reason, the authors most often choose the Ramirez technique, anterior component separation, if there is a need to release the muscle [2].

Anterior component separation is the release of rectus abdominis, transverse abdominis and internal oblique muscles from external oblique muscle insertion just beyond the aponeurosis. The layer between the oblique muscles is practically avascular, which is why dissecting this layer is safe. 
Similarly, intestinal adhesion dissection is carried out without the unnecessary release of patent intestinal loops. In the absence of ileus symptoms prior to the surgery, the authors advise not performing unnecessary adhesiolysis if it does not concern the anterior abdominal wall.

This prevents the possibility of intestinal loops folding on adhesions leading to ileus while releasing the muscle compartments medially. Moreover, avoiding abdominal loop dissection reduces peri-operative trauma and the risk of iatrogenic enterotomy.

In the case of extensive defects (in the transverse dimension) and the lack of a possibility of closing the fascia in the midline, the authors use the bridging technique in order to avoid abdominal compartment syndrome. This method reduces the risk of anastomotic leakage and enhances peristalsis. Similar to the sub-lay technique, the implant covers the defect with a margin (under the rectus muscles) but its central part remains loose, forming "the bridge." The anterior rectus sheath is sewn into the implant with a non-absorbable suture, usually polypropylene 2-0.

Special attention is paid to the choice of the implant type and the surgical implantation technique [3]. The porosity of the mesh is an important factor; the pore size is based on the assumption that bacteria penetrate freely through the microporous mesh (less than $10 \mu \mathrm{m}$ ) and micropores act as a filter on macrophages, preventing effective phagocytosis. For this reason, it is believed that macroporous mesh implantation in a contaminated environment is advisable. Complications after mesh implantation in the contaminated surgical area occur in 10-15\% of cases. These complications include surgical site infection, intestinal fistula and skin necrosis. Proper surgical technique minimizes the risk of complications following mesh implantation into the contaminated surgical field. Bury et al. showed that poor surgical technique and improper mesh fixation that cause waving or folding may promote infection persistence and sometimes leads to the removal of the mesh [4]. It has also been shown that the use of a synthetic mesh in a contaminated field is safe with a proper surgical technique and macroporous implant structure. The structure, rigidity and weight of the implant are also important when closing or bridging the abdominal cavity defect. If reconstruction of the linea alba is possible, the implant flexibil- ity (less mass, greater mesh porosity) may be higher; the implant is only a scaffold for reconstructed anatomic layer augmentation. In the case of bridging, the implant replaces part of the anterior abdominal wall and therefore should have a denser structure to prevent bulging once the inflammation subsides on the mesh.

To assess the blood supply to cutaneo-subcutaneous wound edges, indocyanine green angiography in infrared light was performed. This technique is characterized by high sensitivity and allows identification of ischemic tissues, thereby reducing the incidence of delayed wound healing and the risk of surgical site infections.

In material published by Patel et al., the incidence of postoperative wound healing complications was lower by a factor of two in the group where wound edges were monitored with indocyanine green angiography compared with the control group (42\% vs. 20\%) [5]. Similar results were published by Colavita in a group of patients with complex abdominal wall defects [6]. Redon drains were maintained until day 3 after surgery, reducing the risk of seroma occurrence. After surgery, the patient was awakened and transferred to the Surgery Department. The patient did not require hospitalization in the Intensive Care Unit or a blood transfusion.

\section{Conclusions}

The use of minimally invasive techniques and intraoperative indocyanine green angiography in infrared light enables safe reconstruction of the anterior abdominal wall with simultaneous reconstruction of the gastrointestinal tract, minimizing the occurrence of early postoperative complications.

\section{Conflict of interest}

The authors declare no conflict of interest.

\section{References}

1. Pędziwiatr $M$, Wierdak $M$, Nowakowski $M$, et al. Cost minimization analysis of laparoscopic surgery for colorectal cancer within the enhanced recovery after surgery (ERAS) protocol: a single-centre, case-matched study. Videosurgery Miniinv 2016; 11: 14-21.

2. Heller L, McNichols CH, Ramirez OM. Component separations. Semin Plast Surg 2012; 26: 25-8.

3. Pawlak M, Bury K, Śmietański M. The management of abdominal wall hernias in search of consensus. Videosurgery Miniinv 2015; 10: 49-56. 
4. Bury K, Smietański M, Justyna B, et al. Effects of macroporous monofilament mesh on infection in a contaminated field. Langenbecks Arch Surg 2014; 399: 873-7.

5. Patel KM, Bhanot P, Franklin B, et al. Use of intraoperative in docyanin-green angiography to minimize wound healing complications in abdominal wall reconstruction. J Plast Surg Hand Surg 2013; 47: 476-80.

6. Colavita PD, Wormer BA, Belyansky I, et al. Intraoperative indocyanine green fluorescence angiography to predict wound complications in complex ventral hernia repair. Hernia 2016; 20: 139-49.

Received: 16.12.2017, accepted: 27.03.2018. 